\title{
The ASL-CDI 2.0: An updated, normed adaptation of the MacArthur Bates Communicative Development Inventory for American Sign Language
}

\author{
Naomi K. Caselli ${ }^{1}$ - Amy M. Lieberman ${ }^{1}$ - Jennie E. Pyers ${ }^{1}$ \\ Published online: 16 March 2020 \\ (C) The Psychonomic Society, Inc. 2020
}

\begin{abstract}
Vocabulary is a critical early marker of language development. The MacArthur Bates Communicative Development Inventory has been adapted to dozens of languages, and provides a bird's-eye view of children's early vocabularies which can be informative for both research and clinical purposes. We present an update to the American Sign Language Communicative Development Inventory (the ASL-CDI 2.0, https://www.aslcdi.org), a normed assessment of early ASL vocabulary that can be widely administered online by individuals with no formal training in sign language linguistics. The ASL-CDI 2.0 includes receptive and expressive vocabulary, and a Gestures and Phrases section; it also introduces an online interface that presents ASL signs as videos. We validated the ASL-CDI 2.0 with expressive and receptive in-person tasks administered to a subset of participants. The norming sample presented here consists of 120 deaf children (ages 9 to 73 months) with deaf parents. We present an analysis of the measurement properties of the ASL-CDI 2.0. Vocabulary increases with age, as expected. We see an early noun bias that shifts with age, and a lag between receptive and expressive vocabulary. We present these findings with indications for how the ASL-CDI 2.0 may be used in a range of clinical and research settings
\end{abstract}

Keywords language deprivation $\cdot$ sign language $\cdot$ vocabulary acquisition

A central characteristic of language development during the first years of life is a rapidly growing vocabulary. Vocabulary growth is predicted by differences in the environment (e.g., input) and in the individual (e.g., processing speed and attentional skills), and crucially, vocabulary in turn predicts later language and literacy abilities (Bates et al., 1994; Duff, Reen, Plunket, \& Nation, 2015). For more than 25 years, the MacArthur-Bates Communication Development Inventory (MB-CDI; Fenson et al., 1994), a parent checklist of children's spoken vocabulary and communication skills, has offered great insight into the earliest stages of vocabulary development and has been able to identify children at risk for language delay (Heilmann, Weismer, Evans, Holler, 2005; Sachse \&

Electronic supplementary material The online version of this article (https://doi.org/10.3758/s13428-020-01376-6) contains supplementary material, which is available to authorized users.

Naomi K. Caselli

nkc@bu.edu

1 Wheelock College of Education and Human Development, Boston University, 2 Silber Way, 3rd Floor, Boston, MA 02215, USA
Von Suchodoletz, 2008). Because vocabulary predicts many aspects of language development and is an early emerging linguistic competence, it is an ideal locus of assessment of the early language skills of signing deaf children. To date, the MB-CDI has been adapted to American Sign Language (ASL) to test expressive vocabulary (which we will refer to as the ASL-CDI 1.0; Anderson \& Reilly, 2002), British Sign Language (BSL) to test both receptive and expressive vocabulary (Woolfe, Hermann, Roy, \& Woll, 2010), and Turkish Sign Language (Sumer, Grabitz, \& Küntay, 2017), and an adaptation to Israeli Sign Language is under way (Meir \& Novogrodsky, 2018). We present here an updated ASL adaptation, the ASL-CDI 2.0, that includes initial normative data for both receptive and expressive vocabulary. We also describe ASL receptive and expressive vocabulary tasks that were developed to validate the ASL-CDI 2.0.

Beyond the ability to assess early sign vocabulary in a clinical setting, sign language adaptations of the CDI enable investigation of two broad questions. First, with this type of assessment, researchers can disentangle the language-general from modality-dependent aspects of vocabulary acquisition. The ASL-CDI 1.0 offers a broad picture of a child's lexicon, so it is possible to understand how the structure of the lexicon shapes 
vocabulary acquisition. In many respects, vocabulary acquisition in sign language parallels spoken language vocabulary acquisition. Deaf children's first produced signs are similar to hearing children's first spoken words, reflecting the salient people, places, things, and routines of the child's environment (Anderson \& Reilly, 2002). Further, despite the prevalence of predicates in the ASL input (Fieldsteel, Bottoms \& Lieberman, in press), verbs are not overrepresented in children's expressive vocabulary; if anything, there is a slight noun bias (Anderson \& Reilly, 2002). Longitudinal data from the BSL adaptation of the CDI shows some modest evidence of a vocabulary growth spurt at around 50 signs or between 16 and 19 months (Woolfe et al., 2010). Despite the broad parallels between sign and spoken language, several studies have also examined differences in vocabulary acquisition across modalities with respect to iconicity and phonology (Caselli \& Pyers, 2017; Caselli \& Pyers, 2019; Thompson, Vinson, \& Vigliocco, 2012).

Second, the sign language adaptations of the CDI allow researchers to ask how the critical period of language acquisition interacts with the quantity and quality of language input to shape language learning and attainment. With few exceptions, the brains of humans have evolved to expect early, rich linguistic input. Deaf children are one of the only groups of people who are at risk for not receiving high-quality and consistent linguistic input during the first years of life. The majority of deaf children are born to parents who do not know sign language at the time the child is born (Mitchell \& Karchmer, 2004), and as such are at risk for language deprivation (Hall W., 2017). Comparing language acquisition among deaf children who are and are not at risk for language deprivation offers a unique window into the of role language exposure in language acquisition. Language acquisition is more efficient in early childhood. At the same time, older children may bring more mature cognitive resources to the task of learning language.

Limited exposure to language during early childhood nevertheless has devastating effects on many other aspects of language and cognitive development (Hall et al., 2016; Hall et al., 2017; Hall, Hall, \& Caselli, 2019), so the early identification of children who are not meeting language acquisition milestones is critical (see Henner et al., 2018 for a recent review). Vocabulary is an early emerging linguistic competence; consequently, vocabulary delays may be an early indicator that a child has not received sufficient linguistic input in the first years of life.

At present, in addition to the ASL-CDI 1.0 (Anderson \& Reilly, 2002), there are two other available ASL assessments designed for use in children from birth to five years: the Visual Communication and Sign Language Checklist (VCSL; Simms, Baker, \& Clark, 2013) and the Ski-Hi Language Development Scale (Ski-Hi LDS; Tonelson, 1980). The VCSL and Ski-Hi LDS both offer a broad-strokes picture of a child's early language proficiency. The VCSL is a robust screening tool that provides percentile norms for a wide range of early language and communication skills among deaf children. This tool is particularly helpful in the initial identification of deaf children who are at risk for delays in communication skills and ASL acquisition. The VCSL and the Ski-Hi LDS must be administered by someone who is fluent in ASL and has expertise in ASL linguistics (e.g., the person must know what a "plain verb" is), or has undergone specific training. Unfortunately, the children most at risk for language deprivation are so in part because they do not work with professionals with such sign language expertise. The ASL-CDI 1.0 can be administered without expertise in the linguistic structure of ASL.

The ASL-CDI 2.0 is intended to improve upon the first adaptation in a few specific ways. At the time the ASL-CDI 1.0 was developed, technology was limited such that it would have been impossible to make a video version of the assessment widely available. In lieu of showing parents actual signs, they were shown glosses-English translations of the target ASL signs. Parents who are not fully bilingual in ASL and English may be unfamiliar with the chosen English translation of the targeted sign. Even fluent bilinguals may not know the intended ASL-English mapping, a task made more complicated by the fact that some English words map to multiple ASL signs (e.g., there are several ASL signs that correspond to the gloss PICNIC). ${ }^{1}$ Another gap in the current ASL-CDI is that it was normed for parental reports of expressive vocabulary only; there is no information about children's receptive vocabulary. Further, it was never validated for use with deaf children of hearing parents, so it is unclear whether the assessment could be used with the vast majority of deaf children who are most at risk for language deprivation. Finally, the authors of the ASL-CDI 1.0 note that, although a goal of the tool was to provide age-level norms for ASL vocabulary, the variability of their sample limited their ability to do so. Thus, it is not currently possible to determine whether a child's vocabulary at a given age falls within the expected range.

More than 20 years after the ASL-CDI 1.0 was created, changes in technology have offered the opportunity to consider an update to the original test that allows for the secure online administration of the measure in a bilingual format using both ASL and English, to expand the measure to collect data about both expressive and receptive vocabulary, to make a set of norms available to practitioners, and to take the first steps to identify whether non-native hearing signers can reliably complete the form for their deaf children. As in the other sign language adaptations of the CDI, we selected a normative sample of deaf children learning sign language from their deaf parents. These children learn ASL in environments that are most comparable to the average hearing child learning spoken language, in that their parents are fluent in ASL, and there is no delay before they begin learning ASL. In what follows, we first

\footnotetext{
${ }^{1}$ Conversely, a single ASL sign could map to several English concepts (e.g., FINISH can mean "all done" or "stop it").
} 
describe the composition of the ASL-CDI 2.0, and then describe a validation study in which we compare children's performance on two in-person ASL vocabulary tasks to the ASLCDI 2.0 parental reports. Following this, we present the psychometric properties of the ASL-CDI 2.0, and describe patterns in children's vocabulary composition and development.

\section{Methods}

\section{ASL-CDI 2.0}

\section{Materials}

Item selection Vocabulary section. Our starting point for the development of the ASL-CDI 2.0 was the ASL-CDI 1.0. The ASL-CDI 1.0 data were made available to us via Wordbank (Frank et al., 2017). The ASL-CDI 1.0 included 537 signs in 20 semantic categories (Animals, Vehicles, Toys, Food and Drink, Clothing, Small Household Items, Furniture and Rooms, Outside Things, Places to Go, People, Games and Routines, Action Words, Descriptive Signs, Signs about Time, Pronouns, Question Signs, Prepositions and Locations, Quantifiers, Helping Verbs, and Connecting Signs). Two of the authors of the current study, both native signers, along with one deaf native signing mother with extensive professional expertise working with young deaf children, reviewed and modified the original list of signs as follows: We retained only the signs from the ASL-CDI 1.0 that appeared in the ASL-LEX 2.0 database (Caselli et al., 2017; http://asl-lex.org/), as this is the most complete current inventory of the ASL lexicon. The videos for each item were taken from ASL-LEX. The result of aligning the ASL-CDI items with ASL-LEX meant the removal of all but a few fingerspelled items (e.g., BUS was retained). We removed a few culturally outdated signs (e.g., CALL-VIA-TTY, TTY), homophonous signs (VITAMINS, VANILLA), and signs that are likely to vary greatly in frequency depending on characteristics that may be related to language acquisition (HEARING_AID presumably is more frequent for children who wear them). Finally, we identified items for which the given English gloss did not correspond to one and only one possible sign, and we subsequently removed these items from the ASL-CDI. These included signs that had significant dialectal variation (e.g., PICNIC, BIRTHDAY, CIRCUS, HALLOWEEN, PIZZA). We compensated for the deletions by adding 24 items. Because there is a link between language deprivation and mental-state understanding (see Pyers \& de Villiers, 2013 for a review), the additions included three mental-state terms (e.g., REMEMBER, UNDERSTAND, MISUNDERSTAND). We added some body part signs in which the sign did not simply involve pointing to the body part (e.g., STOMACH). The modification process yielded a final set of 533 items, including 274 nouns, 127 verbs, 62 adjectives, 10 adverbs, 2 numbers, and 58 function words, as categorized in ASL-LEX. The items were put into semantic categories largely following the categories laid out in the ASL-CDI 1.0 (see Table 1).

Gestures and Phrases section. The ASL-CDI 1.0 did not include sections on gestures or phrases. We translated items from the gestures portion of the English version of the MBCDI Words and Gestures form into ASL. We excluded items that may not be relevant to deaf children (e.g., questions about singing or routines like "this little piggy" and "so big" that are not commonly played in ASL). The final form included the following sections: Phrases $(n=24)$, First Communicative Gestures $^{2}(n=9)$, Games and Routines $(n=8)$, Actions with Objects $(n=30)$, Pretending to be a Parent $(n=31)$, and Imitating Other Adult Actions $(n=26)$.

All of the non-identifiable data reported here is available, and none of the experiments was preregistered.

\section{Administration}

Data for this study were collected via Survey Monkey. All instructions, questions, and answer choices were presented in ASL and English. The assessment was divided into four sections: three sections of vocabulary, and one section for the Gestures and Phrases. Signs were grouped by semantic category. Form A included the categories Action Signs, Animals, Connecting Signs, Helping Verbs, Question Signs, Toys, and Vehicles; Form B included the categories Body Parts, Clothing, Descriptive Signs, People, Pronouns, and Small Household Items; and Form $\mathrm{C}$ included the categories Food and Drink, Furniture and Rooms, Games and Routines, Mental State Terms, and Outside Things and Places to Go. The order of the items within the categories was randomized, and the order of the three survey sections was counterbalanced. The Gestures and Phrases form was always administered last. Although items were presented only in ASL, parents could also click a link to see an English gloss of the sign, in case they were unfamiliar with the sign and/or to disambiguate it from other signs (e.g., homophones). Out of concern that some items may have substantial dialectal variation that researchers did not identify during the item modification phase, parents could also indicate that their family used a different sign, or that they did not know the sign. We included this final option so that the ASLCDI 2.0 can ultimately be used by hearing parents who may not be familiar with all of the signs on the checklist. Parents were instructed to indicate that a child knew the sign even if the child did not use the "correct" adult sign (e.g., produces the sign with a phonological error).

\footnotetext{
${ }^{2}$ We use the term "gesture" in keeping with the MB-CDI, but in some cases there is not a clear distinction between a gesture and a sign (e.g., pointing at an interesting object).
} 
Table 1 Number of signs by semantic category

\begin{tabular}{ll}
\hline Semantic Category & Number of Signs \\
\hline Action Signs & 112 \\
Animals & 35 \\
Body Parts & 15 \\
Clothing & 23 \\
Connecting Signs & 5 \\
Descriptive Signs & 57 \\
Food and Drink & 45 \\
Furniture and Rooms & 19 \\
Games and Routines & 18 \\
Helping Verb & 2 \\
Mental State Terms & 23 \\
Outside Things and Places to Go & 32 \\
People & 28 \\
Prepositions and Locations & 16 \\
Pronouns & 13 \\
Quantifiers & 13 \\
Question Signs & 10 \\
Signs About Time & 15 \\
Small Household Items & 38 \\
Toys & 6 \\
Vehicles & 8 \\
\hline
\end{tabular}

Sampling and participants We recruited families to participate in the normative data sampling by asking parent-infant programs at schools for the deaf with an ASL-English bilingual philosophy to distribute a recruitment notice. In addition, we reached parents through a Facebook advertisement and through word of mouth. We relied heavily on snowball sampling to recruit many of our participants. Because recruitment was almost exclusively online, we confirmed parent's knowledge of ASL by requiring the parent who was completing the ASL-CDI to complete a three-question ASL vocabulary check before receiving subsequent links to the survey.

Primary normative sample We collected ASL-CDI 2.0 reports for 145 deaf children who had at least one deaf parent. Twenty-five children $\left(M_{\text {age }}=29\right.$ months; range $\left.=11-67\right)$ whose parents reported additional diagnoses related to language acquisition, e.g., autism spectrum disorder $(n=9)$, and/or were blind or low-vision $(n=12)$ plus parents who did not respond to either of these questions $(n=6)$ were excluded from all subsequent analyses. The final sample for the normative data included 120 participants ( 58 females and 62 males) from 26 states in the United States and two provinces in Canada. The ethnic and racial breakdown of the normative sample was 17 African American/Black, one Asian, one Native American/Alaskan, 89 White, plus five people who identified with more than one race, and nine who did not respond. Nine participants were Hispanic or Latino, 104 were not, and seven did not respond.

Longitudinal sample Of the 120 unique participants, a subset participated more than once to provide data on vocabulary growth over time. Six children participated three times, and 27 participated twice. Because of the length of the test, the repeat participants did not complete the ASL-CDI Gestures and Phrases portion of the test.

Because Anderson and Reilly (2002) reported that their sample did not reach ceiling on the ASL-CDI 1.0, and because we wanted the ASL-CDI 2.0 to be able to be used with older language-deprived children, we intentionally recruited a larger age range than is typical for most adaptations of the CDI. The average age of the final normative sample was 31.98 months (median = 29; range 9-73 months; see Fig. 1). Half of the children had a primary caregiver who had at least a college degree $(n=67), 38$ either a high school degree or some college completed, and 15 with no high school degree. All families reported using ASL in their homes.

Parents were given a $\$ 25$ gift card for each of the three sections of the vocabulary checklist and a $\$ 15$ gift card completion bonus for completing the entire assessment (a total of up to \$90). They received no additional compensation for the Gestures and Phrases section. Parents did not have to complete the entire checklist in one sitting, but we did require that they complete all surveys within one week. Perhaps due to the length of the assessment - the average time to complete all three of the sections of the vocabulary checklist is estimated at an hour and 45 minutes - 20 parents skipped more than 10 vocabulary items ( $M=51.4$ skipped items). Given that the order of surveys was counterbalanced across participants, and the order of the items within each semantic category was randomly presented, the missing data was equivalently distributed across items. As such, incomplete reports were included in the analysis (removing items that were skipped), and vocabulary size was computed as a proportion of the total items for which parents entered a response. Additionally, parents indicated that their families did not use some signs (see Supplementary A for a list) or did not know some signs. By scoring with a proportion rather than a raw number, we were able to remove these signs from the scores so that items with dialectal variation did not count against the children's vocabulary.

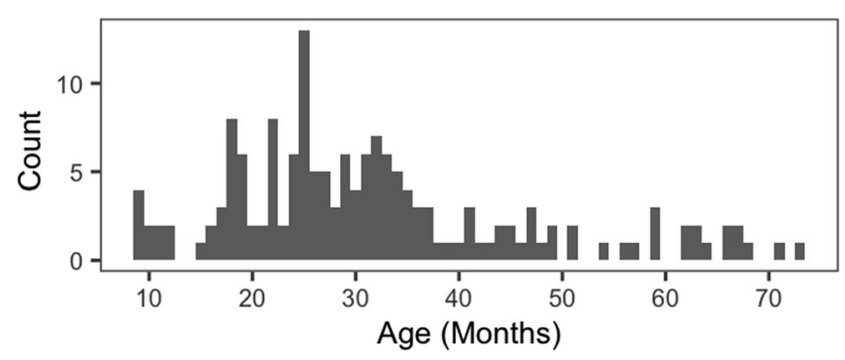

Fig. 1 Distribution of ages in the normative sample 


\section{$A S L-C D I 2.0$ validation study}

We developed two direct in-person assessment tasks to validate the ASL-CDI 2.0 for use with deaf children with deaf parents and to probe whether hearing parents could report their child's sign language vocabulary abilities. One was a picture-matching task that measured receptive vocabulary skills by asking participants to view a sign and pick the matching picture from an array of four pictures (Fig. 2, left). The other was a picture-naming task that measured expressive vocabulary knowledge by asking participants to produce the sign for a pictured item (Fig. 2, right). All of the items on the picture-naming and picture-matching tasks appear on the ASL-CDI 2.0.

\section{Validation study participants}

A subset of the normative sample $(n=29$; mean age $=44$ months, range $=22-68$ months) completed the two in-person vocabulary measures within one week of completing the ASL-CDI 2.0. We also recruited 11 non-native signing hearing parents with deaf children between the ages of 24 and 67 months ( $M=45$ months) to complete the ASL-CDI 2.0 for their deaf children and to have their child complete the inperson tests. Families were paid $\$ 25$ for the in-person visit, and children were given a small toy and a book.

\section{Validation task}

Item selection Items for the expressive and receptive vocabulary tasks were selected from the subset of ASL-CDI 1.0 expressive vocabulary reports from Anderson and Reilly (2002) that were reported in Caselli and Pyers (2017) and are available at https://osf.io/uane6/. To be sure that the items on the test reflected different degrees of difficulty, we divided ASLCDI 1.0 reports into four age bins $(7-14,14-22,22-28$, and 28-36 months). For each sign, we identified the proportion of children in an age bin that could produce the sign $(N$ produced/Total $N$ ). We excluded signs that did not have clear increasing acquisition patterns (e.g., some young children knew the sign, but older children did not), signs that were not easily picturable (e.g., COUSIN), lexicalized fingerspelled signs (e.g., TV), and compound signs (e.g., PLAYGROUND). We then identified the minimum age group at which at least $50 \%$ of the children knew the sign in order to select 16 signs for each age bin: four targets for each of two forms, plus two foils for each of four targets (foils could serve as targets on both forms, so the target from one form always served as a foil on the other form). The youngest age bin (7-14 months) did not have enough items that met these conditions, and was shy by 12 items. We selected the items that the most children in the 7-14-month-old age range had acquired (range 18-64\%, $M=$ $38.4 \%, S D=35.0 \%$ ), as long as the next bin up had a greater acquisition rate. We expected the production data from the ASL-CDI 1.0 to underestimate age of acquisition of receptive vocabulary; thus we added a $>36$ category so that we had items that would be challenging for children across the entire target age range. Items in the $>36$ months category had to have been acquired by less than $50 \%$ of the children in the 28-36 bin, but more than $0 \%$ (range $33-48 \%, M=35.2, S D=33.9$ ). The best-fitting items (i.e., those with the largest increase from the prior age bin, or those that did not have multiple possible variants in ASL) were selected as targets, and assigned to one of two forms. We balanced the two forms as much as possible with respect to semantic category (e.g., roughly equal numbers of foods, animals on both forms for each category). We also tried to keep closely semantically related items on separate forms (e.g., MOP and SWEEP).

Foils on the picture-matching task always came from the same age bin as the target. Because the targets were the bestfitting items, they always served as foils on the alternate form. Foils were selected to evenly distribute semantic categories so that, as much as possible, each item had one foil that was semantically similar, but not all of the foils came from the same semantic category as the target (e.g., a food might have one food as a foil but not three). Foils that formed minimal
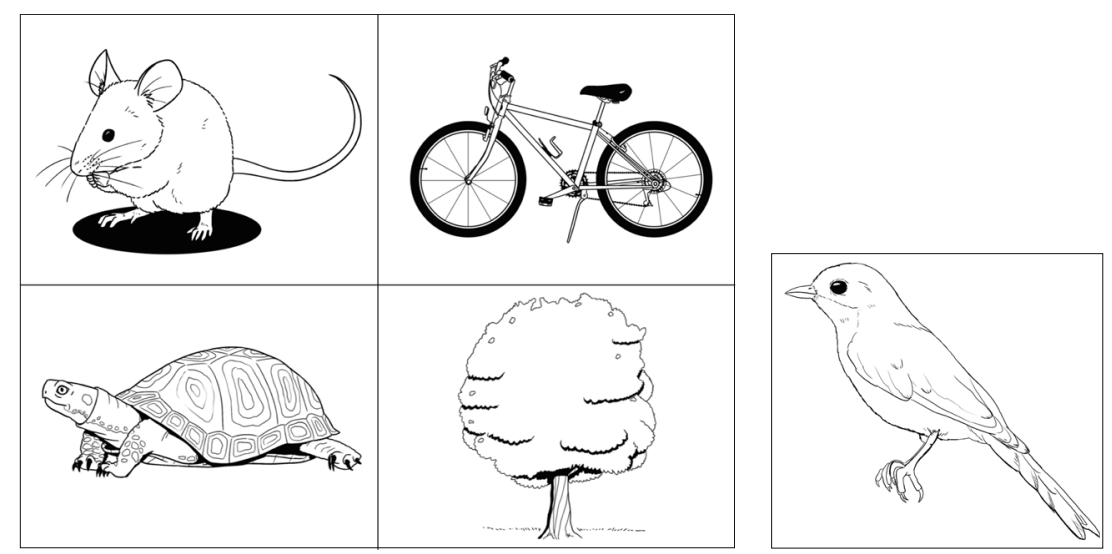

Fig. 2 Sample item on the picture-matching task (left) and picture-naming task (right). The same illustrations were used in the picture-naming task. In this case, the target for the picture-matching question was TREE, and for the picture-naming question was BIRD 
pairs or close minimal pairs with the target were avoided, as were signs that were highly semantically related (SODA and BOTTLE). Foils that had both phonological and semantic overlap with the target were avoided (e.g., GIRAFFE and FROG are both animals and both use the neck location). We also tried to evenly distribute the number of visually complicated images across the forms and items. A handful of foils had multiple possible sign variants (e.g., pictures of corn, cake, a rabbit, and a strawberry each correspond to multiple possible signs), but these items were not used as targets.

Pictures were illustrated by a deaf native signing artist (see Fig. 2). All pictures were in black and white, and designed to be relatively similar with respect to visual interest and complexity. Four native signing adults viewed each picture and produced the matching sign. Only pictures that elicited $100 \%$ naming accuracy for the target sign were included in the test.

Items were presented in pseudorandomized order (four randomly selected item orders for each of the two forms of the test). We did this psuedorandomization in the event that if some children only partially completed the test, each item had roughly the same number of responses. The 20 items on each test were divided into four blocks of five, and each block had one item from each age category $(7-14,14-22,22-28,28-36$, and >36). This way, the youngest children would see a mix of items that were likely to be in their vocabularies (i.e., so they would not be discouraged by a series of items they were unlikely to know). The order of the blocks, the items drawn from each age category, and the order of the items in the blocks were randomly generated. The placements of the target and foils on each page of the picture-matching test were randomized.

The picture-matching and picture-naming tasks had identical targets.

Procedure The first item was always a practice item, and children were given feedback on this item. Otherwise, no feedback was given. The instructions for the tests were as follows:

Picture-matching: For the first item, say SEE [point to all four pictures]. WHERE [ITEM]? Sign the target item using the variant that is in the booklet. For following items, just say WHERE [ITEM]. If the child does not respond, point to each picture in turn and ask a yes/no question: [ITEM]? (e.g., is this the baby?).

Picture-naming: Show the child the picture, and say WHAT [point to picture].

Participants completed different forms for each vocabulary test.

Coding The picture-matching responses were recorded as correct if the child pointed to the picture in the array that matched the experimenter's sign. All other responses were scored as incorrect.

Picture-naming responses were scored by deaf native signers. The responses were considered correct if the participant produced the expected sign. Responses did not need to be adult-like productions of the signs so long as the native signing coder could recognize the intended sign. Phonological and inflectional variants were also considered correct if the coder recognized the sign as the intended sign (e.g., DUCK with two versus four fingers). If the child produced a sentence or multiple answers, the response was considered correct if the target item appeared in the sentence or among the answers. Items were considered incorrect if the participant said they didn't know the sign, provided no response, provided an unrelated response, or provided a superordinate or subordinate category (e.g., FOOD for a picture of salad).

\section{Results}

We first examined the concurrent validity of the ASL-CDI 2.0 by (1) comparing the Vocabulary section to the vocabulary elicited in the validation study (picture-naming and picturematching; $n=40$ ) and (2) comparing the scores from the entire normative sample $(n=120)$ on the Vocabulary section to the Gestures and Phrases section. Having established validity, we then report findings from the full normative sample regarding the size and composition of ASL vocabulary in deaf children. Finally, we describe specific applications for practitioners using a short-form version of the ASL-CDI 2.0 and a newly developed parent portal for the administration of the ASL-CDI 2.0 .

\section{Concurrent validity}

ASL-CDI 2.0 Vocabulary compared with picture-naming and picture-matching Deaf parents $(n=29)$ were highly reliable reporters of their children's vocabularies. The ratio of items that children correctly produced on the picture-naming test to the same items the parents reported the child could produce on the ASL-CDI 2.0 was $75.3 \%$. For children's receptive vocabulary, the mean agreement was $83.6 \%$. Hearing parents $(n=$ 11 ), despite being generally newer signers, were comparably reliable reporters of their children's vocabularies (productive $=$ $69.3 \%$, receptive $=77.3 \%$ ). Deaf and hearing parents' accuracy was compared using the Wilcoxon rank-sum test due to the sample size and non-normal distribution. Parents in the two groups did not differ in how accurately they represented their children's expressive $(W=221.5, p=0.12)$ or receptive vocabularies ( $W=227, p=0.07)$. Figure 3 illustrates the accuracy in reporting in the two groups. 

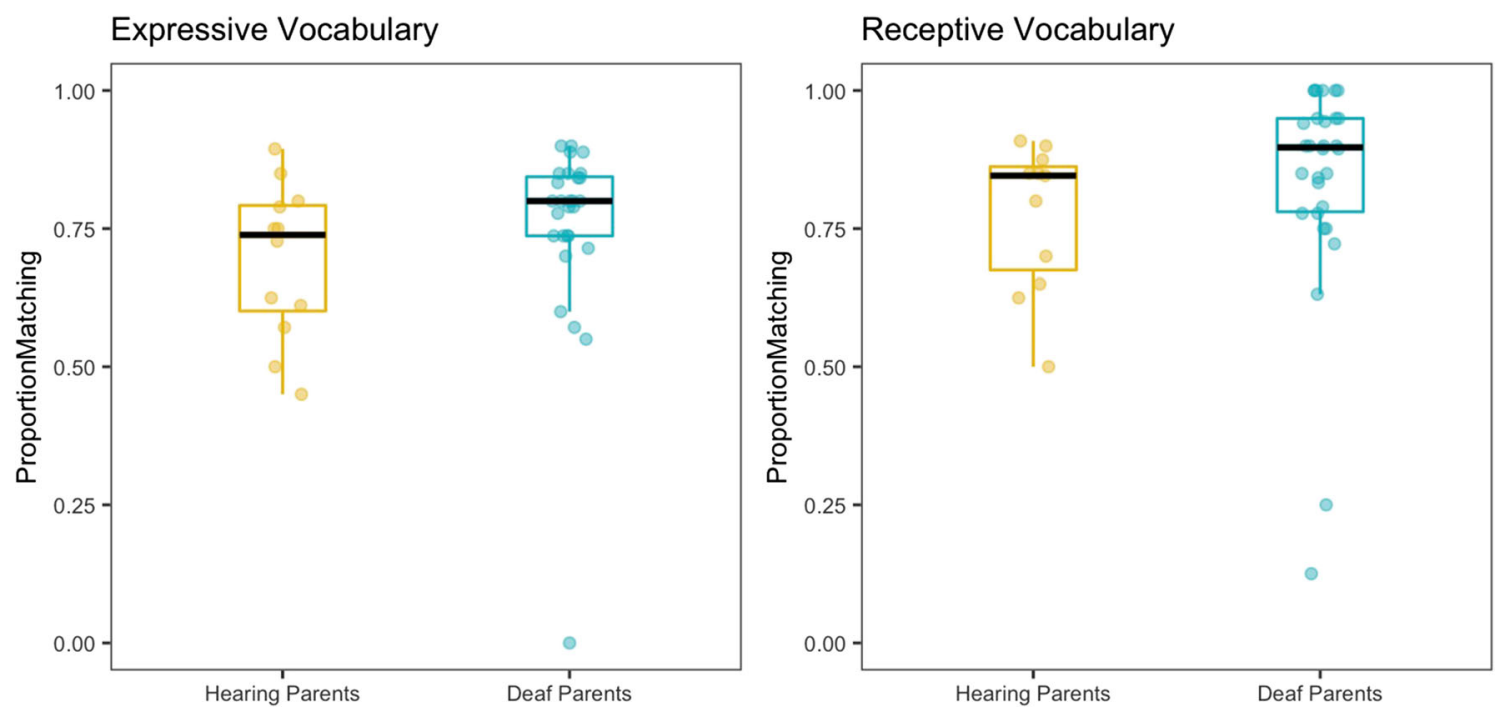

Fig. 3 The proportion of signs on the in-person test that the parent accurately reported on the ASL-CDI 2.0

ASL-CDI 2.0 vocabulary compared with Gestures and Phrases Expressive vocabulary size was related both to the number of gestures a child produced and to the number of phrases a child understood (Fig. 4). Children could frequently understand many common phrases and could engage in gestural routines even when they had few or no signs in their vocabularies. The reverse was not the case; rather, children who knew many signs could almost always understand common phrases and engage in gestural routines.

\section{Assessment norms}

Vocabulary The relationship between vocabulary size and age is plotted in Fig. 5. The ASL-CDI 2.0 detects expected effects of age on vocabulary size in deaf native signing children who have no additional diagnoses related to language acquisition, with older children having larger vocabularies than younger children. Children hit ceiling by around 40 months on the receptive portion and by 60 months on the expressive portion of the assessment. Many other adaptations of the MB-CDI are designed for children younger than these ages, although no child in the under-36-months samples reported with the ASLCDI 1.0 and the BSL-CDI reached ceiling performance. By collecting data from older children, we see more clearly the different, albeit expected, acquisition patterns for receptive and expressive vocabulary, whereby expressive vocabulary develops more slowly than receptive vocabulary. The extended age range may in part be because the ASL-CDI 2.0 has more mental-state vocabulary items (including CRAZY, DISAPPOINT, MISUNDERSTAND, and THINK), and these
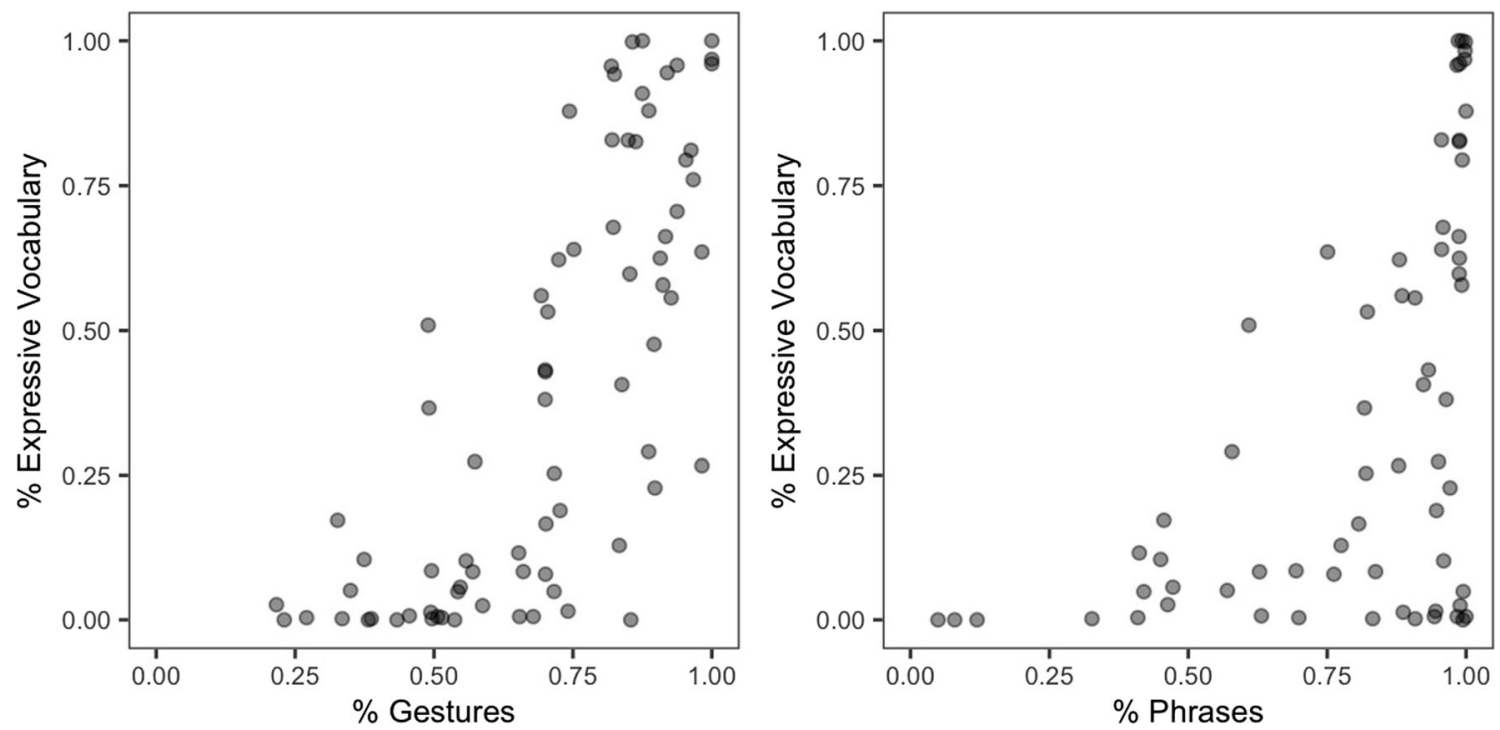

Fig. 4 The relationship between expressive vocabulary size (proportion of signs the child can produce) and the proportion of gestures they produce (left) and phrases they understand (right). The gestures reflect an average of all five sections on the gestures form 


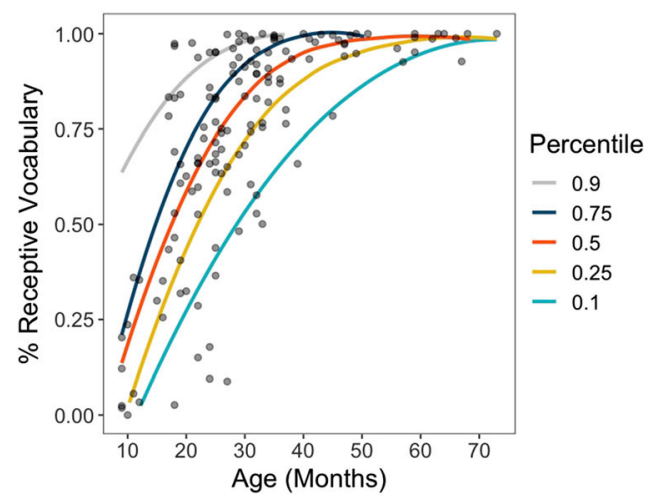

Fig. 5 The relationship between the proportion of signs children were reported as knowing and their ages. Lines indicate the 10th, 25th, 50th, 75 th, and 90th percentiles, and were generated using the gcrq function in the package quantregGrowth. Individual dots represent a single ASL-CDI

were among the signs that were consistently not yet produced by children older than 36 months (among other abstract signs like COUNTRY, PENNY, and EACH). In the ASL-CDI 2.0 online interface, described in more detail below, we provide practitioners with estimates of a child's performance relative to the normal range (above or below the 16th percentile).

Longitudinal data Children's vocabulary growth over time corresponds well with the cross-sectional normative data (see Fig. 6). These data reveal an expected sharp increase in vocabulary size, particularly expressive vocabulary.

Gestures and Phrases The relationship between deaf children's ages and the proportion of gestures they produce and phrases they understand is plotted in Fig. 7. The ASL-CDI 2.0 detects expected developmental curves in both Gestures and Phrases in deaf native signing children who have no additional diagnoses related to language acquisition. There is more variability in the youngest children in the sample on this portion of the test than on the vocabulary section. Development levels off by around 30 months for both Gestures and Phrases. Again, in the ASL-CDI 2.0 online interface, we provide practitioners

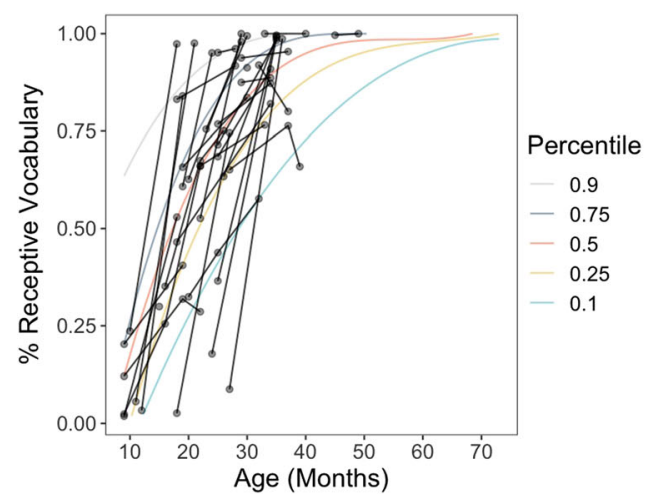

Fig. 6 The relationship between the proportion of signs children were reported as knowing and their ages over time. The colored lines are the same as those in Fig. 5, and indicate the 10th, 25th, 50th, 75th, and 90th

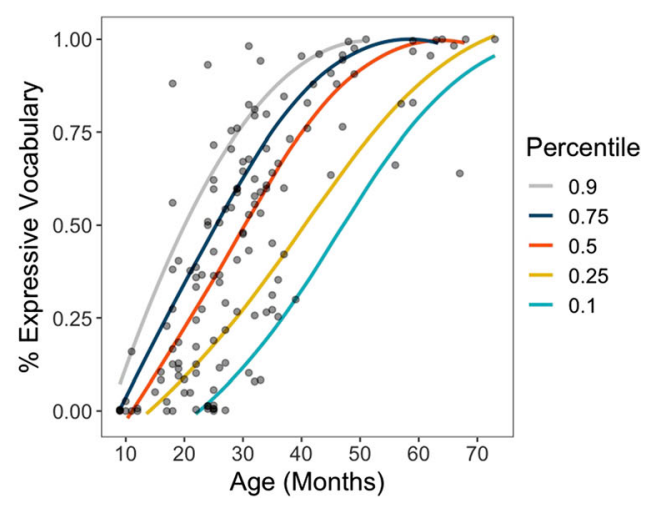

2.0 report (longitudinal data are reported here). These graphs are based on a different set of calculations from the reference levels, and are not intended to be used to classify children's vocabularies as within/above/ below the normal range

with estimates of a child's performance relative to the normal range (above or below the 16th percentile).

\section{Vocabulary composition}

Age of acquisition (AoA) We calculated the age at which each sign is expected to be acquired in two ways. The first was to identify the minimum age (rounded to whole months) at which all children in an age group can produce the sign (e.g., Dale \& Fenson, 1996). However, despite our relatively large sample size compared with previous studies of ASL acquisition, the current data set is quite sparse for this method. The small numbers of children in each age group may lead to greater error in these estimates. Therefore, we adopted a second approach that used the Bayesian generalized linear model (GLM) to determine age of acquisition (e.g., Frank et al., 2019). The two AoA estimates were highly correlated $(r=0.82, p<0.01)$. Both the empirical and Bayesian GLM estimates for each item are presented in Supplementary B. All but one of the first ten words in this data set appear on the list of first 35 signs reported by Anderson and Reilly (2002). Another noteworthy pattern was that the sign MILK was often acquired earlier than other signs

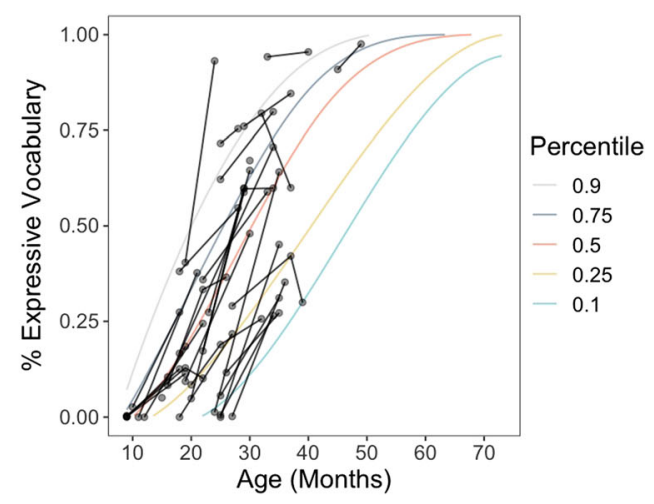

percentiles. Each grey dot represents a single ASL-CDI 2.0 report, and the black lines connect the reports corresponding to a single child to illustrate the child's growth in vocabulary over time 


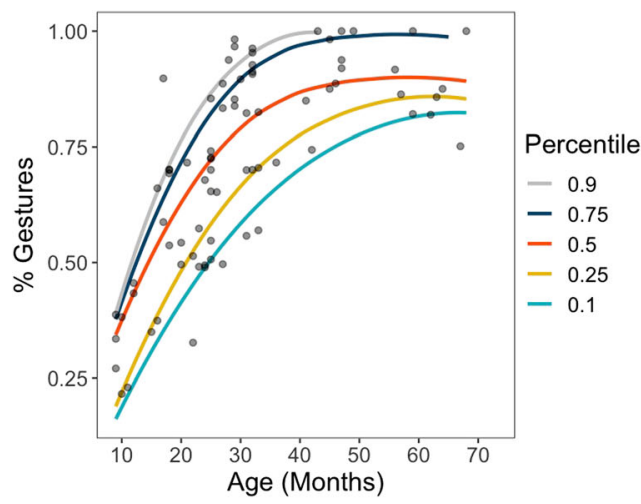

Fig. 7 The relationship between the proportion of Gestures and Phrases children were reported as knowing to their ages. Lines indicate the 10th, 25th, 50th, 75th, and 90th percentiles, and were generated using the gcrq function in the package quantregGrowth. Individual dots represent

(around 10 months of age), with other early-learned signs being acquired a bit later (16-18 months of age).

Semantic categories We next asked whether children were more or less likely to know words in each semantic category. If children are no more likely to know signs of a category than by chance, then the proportion of signs the child knows in that category should be perfectly correlated with the proportion of signs the child knows on the entire assessment (i.e., a child who knows $50 \%$ of the words on the ASL-CDI 2.0 should also know $50 \%$ of the animals). Chance is represented by the black diagonal lines in Fig. 8. If children are more likely to know words in the category than would be expected by chance, the bulk of the distribution should fall above the diagonal line, and if children are less likely to know words in the category than would be expected by chance, the bulk of the distribution should fall below the diagonal line. The patterns here largely resemble those of children acquiring many other languages: animals, toys, and vehicles are overrepresented, and signs about time, quantifiers, and connecting signs are underrepresented (Frank et al., 2019).

Lexical categories In the same way, we compared the proportion of all signs on the assessment that the child could produce to the proportion of signs in a specific lexical category that the child could produce (Fig. 9, left panel). If there was no bias, these proportions should be perfectly correlated (represented by the black diagonal line in Fig. 9). We found limited evidence for a weak early noun bias (represented by the yellow line) and a weak early predicate anti-bias (represented by the red line). The visualization shows that children with smaller vocabularies produce relatively more nouns than would be expected given the proportion of nouns on the ASL-CDI 2.0. At the same time, they produce relatively fewer predicates than would be expected. As children's vocabularies grow, there is a shift in their bias, largely driven by an increase in knowledge of predicates. In addition, we find that, as in most

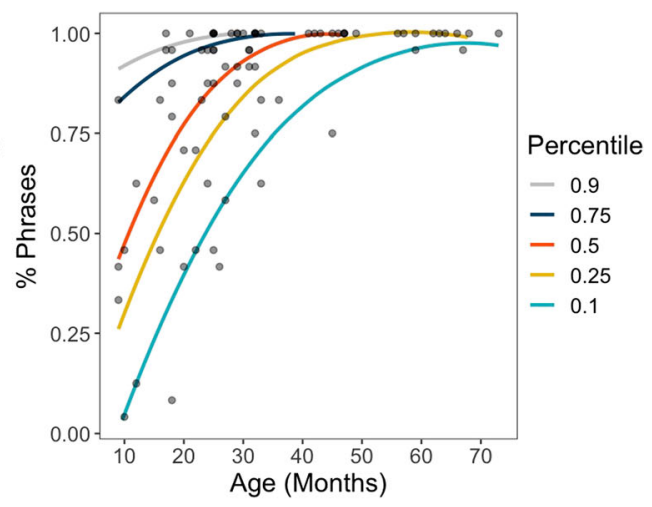

individual children. These graphs are based on a different set of calculations from the reference levels, and are not intended to be used to classify children's performance as within/above/below the normal range

languages, function words are underrepresented (represented by the blue line; Fig. 9). These findings correspond with Anderson and Reilly (2002), who also report a noun bias in early ASL vocabulary, noting that the noun-to-predicate ratio diminishes with age. To confirm these parallel results, we replotted the ASL-CDI 1.0 data that was reported in Caselli and Pyers (2017), and found a similar pattern in both direction and magnitude (Fig. 9, right panel).

\section{Using the ASL-CDI 2.0}

Short form Viewing all 533 videos is time-consuming for parents. We asked whether a short form of the test might provide a similar picture of the child's vocabulary skills. For each child whose form included at least 500 items (i.e., excluding incomplete forms or forms with many items the family did not use; $n=69$ ), we compared the proportion of items they knew of the entire set of items on the ASL-CDI 2.0 to a randomly sampled subset of items from the inventory without replacement. We repeated this procedure 10 times for each child. We did this for four possible subsets, a vocabulary size of 10,30,100, and 500 signs. Figure 10 shows the relationship between scores on the entire test and scores on the subset. Results indicate that scores on a subset of 30 items are highly correlated with receptive scores $(r=0.98, p<0.001)$ and expressive scores $(r=0.99$, $p<0.001$ ) from the full set of items. The efficacy of a short form for the ASL-CDI 2.0 is consistent with work on several other adaptations of the MB-CDI (Mayor \& Mani, 2018).

\section{Accessing the ASL-CDI 2.0}

We developed an online portal where researchers and other professionals who work with deaf children can complete the ASLCDI 2.0 (https://www.aslcdi.org). Parents can sign up for an account in the portal and complete a consent form that allows for the sharing of their child's de-identified data with researchers. Parents fill out a demographic questionnaire that 

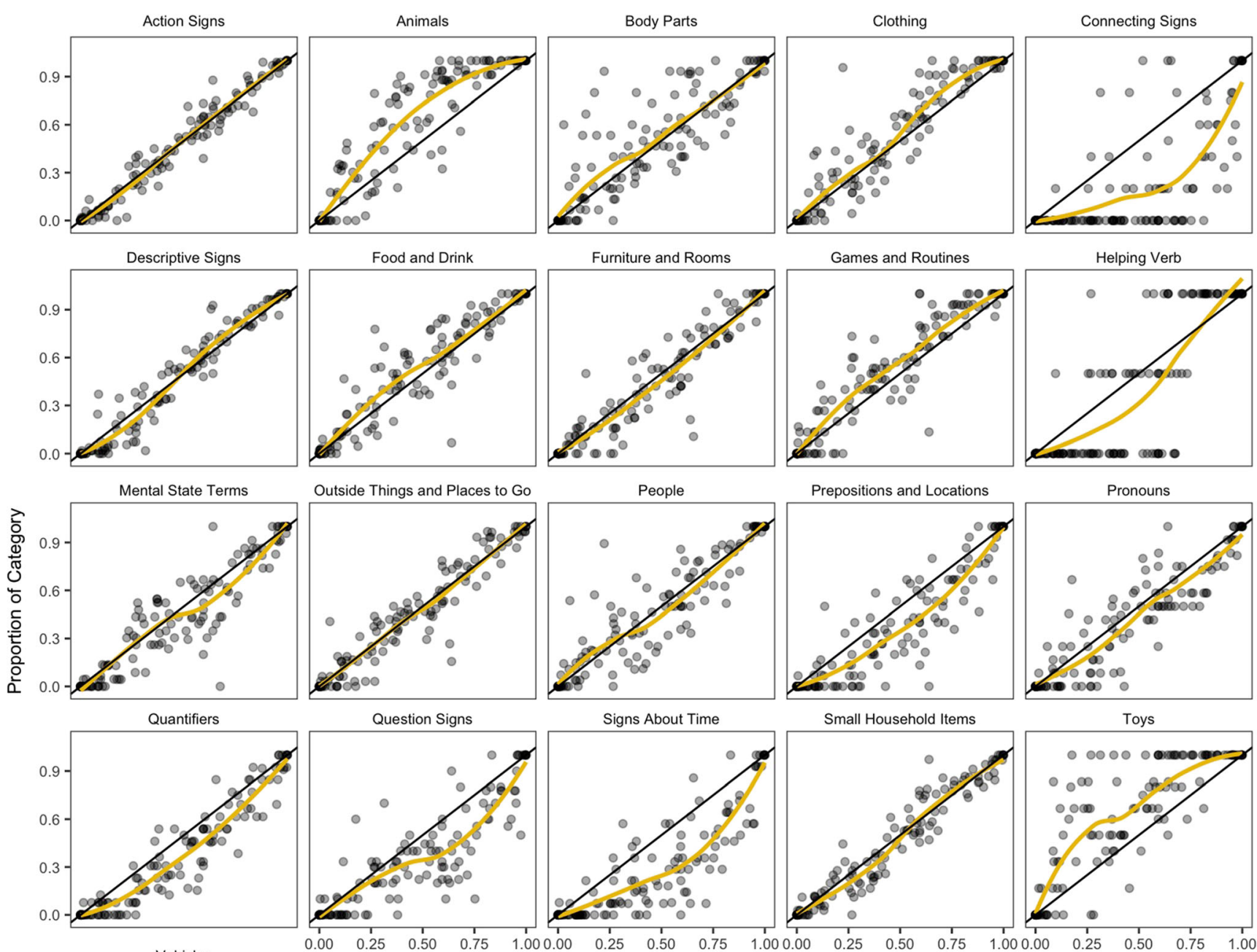

Small Household Items
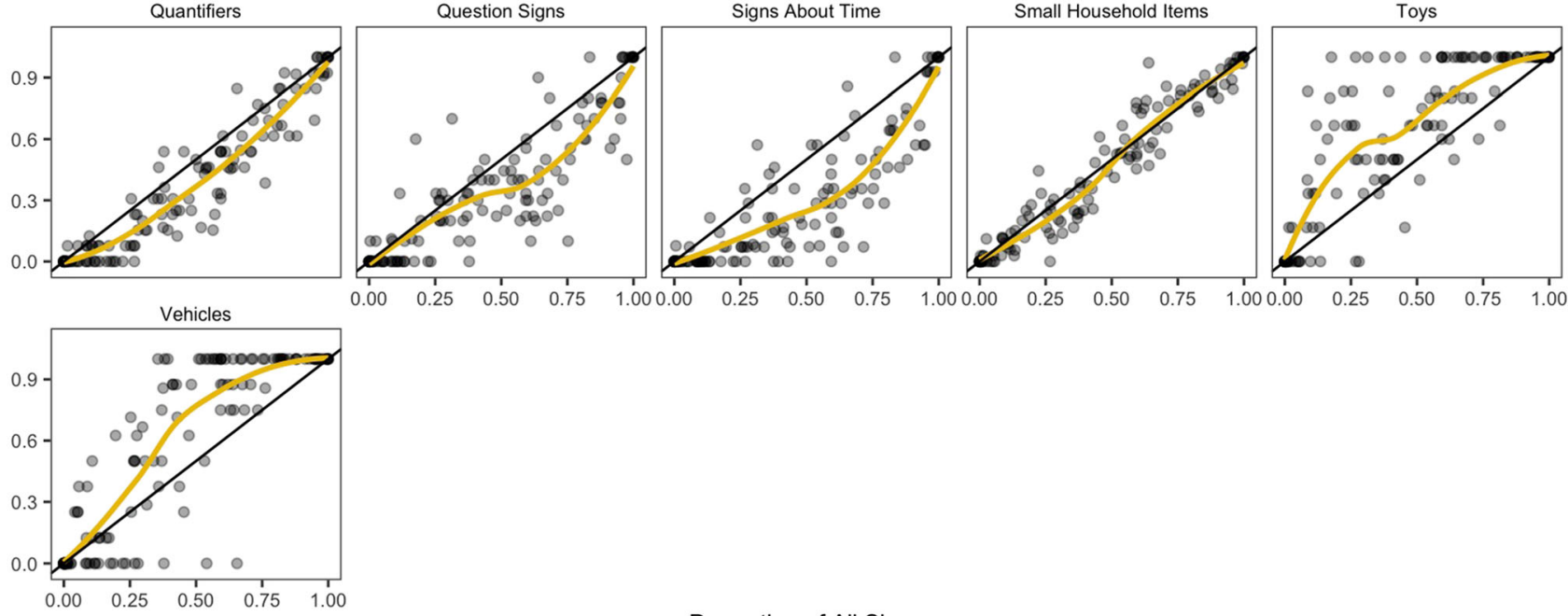

Proportion of All Signs

Fig. 8 The proportion of all the signs on the ASL-CDI 2.0 in a child's expressive vocabulary relative to the proportion of signs of a particular semantic category in a child's expressive vocabulary. Black diagonal line indicates the expected relationship if there were no bias for or against that category. Yellow lines that fall above the black line indicate that this category is overrepresented in children's vocabularies, and yellow lines that fall below the black line indicate that this category is underrepresented in children's vocabularies. Individual dots represent individual children. Plots and analysis technique were modeled after Frank et al. (2019) includes questions about their child's language experience. They can then choose to complete either the comprehensive form of the ASL-CDI 2.0 or a short form that consists of 30 vocabulary items randomly selected from the comprehensive form. Parents then view videos of each sign, and indicate whether their child does not know, understands, or understands and produces each sign (or if they use a different sign, or do not know the target sign). After completing the survey, parents can view a summary report of their child's vocabulary, Gestures and Phrases. Parents also have the option to share their report with an organization (e.g., a research team, school, or early intervention specialist). Organizations can sign up for an account where they can view all of the reports that have been shared with them. The portal also contains information about each child's vocabulary relative to the normal range. Parents can opt in to contribute their child's data to the normative sample, and the norms used to calculate a child's percentile on the portal will be periodically updated to reflect this growing sample. 


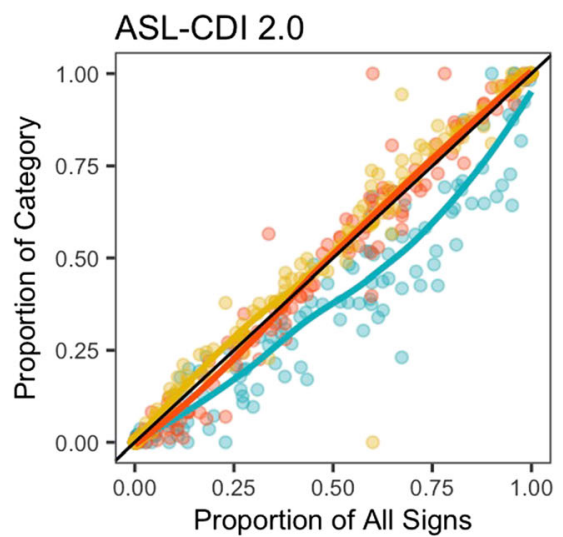

Fig. 9 The proportion of all the signs on the ASL-CDI in a child's expressive vocabulary relative to the proportion of signs of a particular syntactic category in a child's expressive vocabulary. Black diagonal line indicates the expected relationship if there were no bias for that category. The plot on the left is the data from the current study, and the plot on the right is the ASL-CDI 1.0 from Anderson \& Reilly (2002) as reported by Caselli and Pyers (2017). The patterns in the two data sets are the same.

\section{Discussion}

In the current study, we developed and tested the ASL-CDI 2.0. The ASL-CDI 2.0 is a valid measure of early ASL vocabulary. There is a high level of concurrent validity between the vocabulary section of the ASL-CDI 2.0 and in-person measures of children's vocabulary, indicating that parents can reliably report their children's vocabularies. This is true both for deaf parents, and in our pilot sample for hearing parents. There is also a high level of

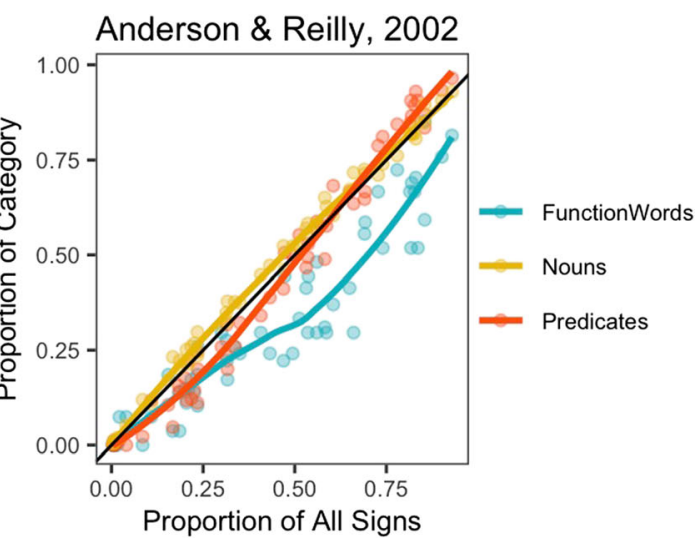

The yellow lines fall slightly above the black lines, indicating that nouns are slightly overrepresented in children who have small vocabularies. The red lines fall slightly below the black lines, indicating that verbs are slightly underrepresented in children who have small vocabularies. The blue lines fall below the black lines, indicating that function words are underrepresented in children's vocabularies. Plots and analysis technique were modeled after Frank et al. (2019)

concurrent validity between the Vocabulary and Gestures and Phrases sections of the ASL-CDI 2.0. The composition of early vocabulary matches that of other languages with respect to both lexical and semantic categories. As expected, children's expressive vocabularies generally lag behind their receptive vocabularies. The first acquired signs are consistent with previously reported early signed vocabularies (Anderson \& Reilly, 2002). Together, these findings suggest that the ASL-CDI 2.0 is a robust instrument for assessing early vocabulary.
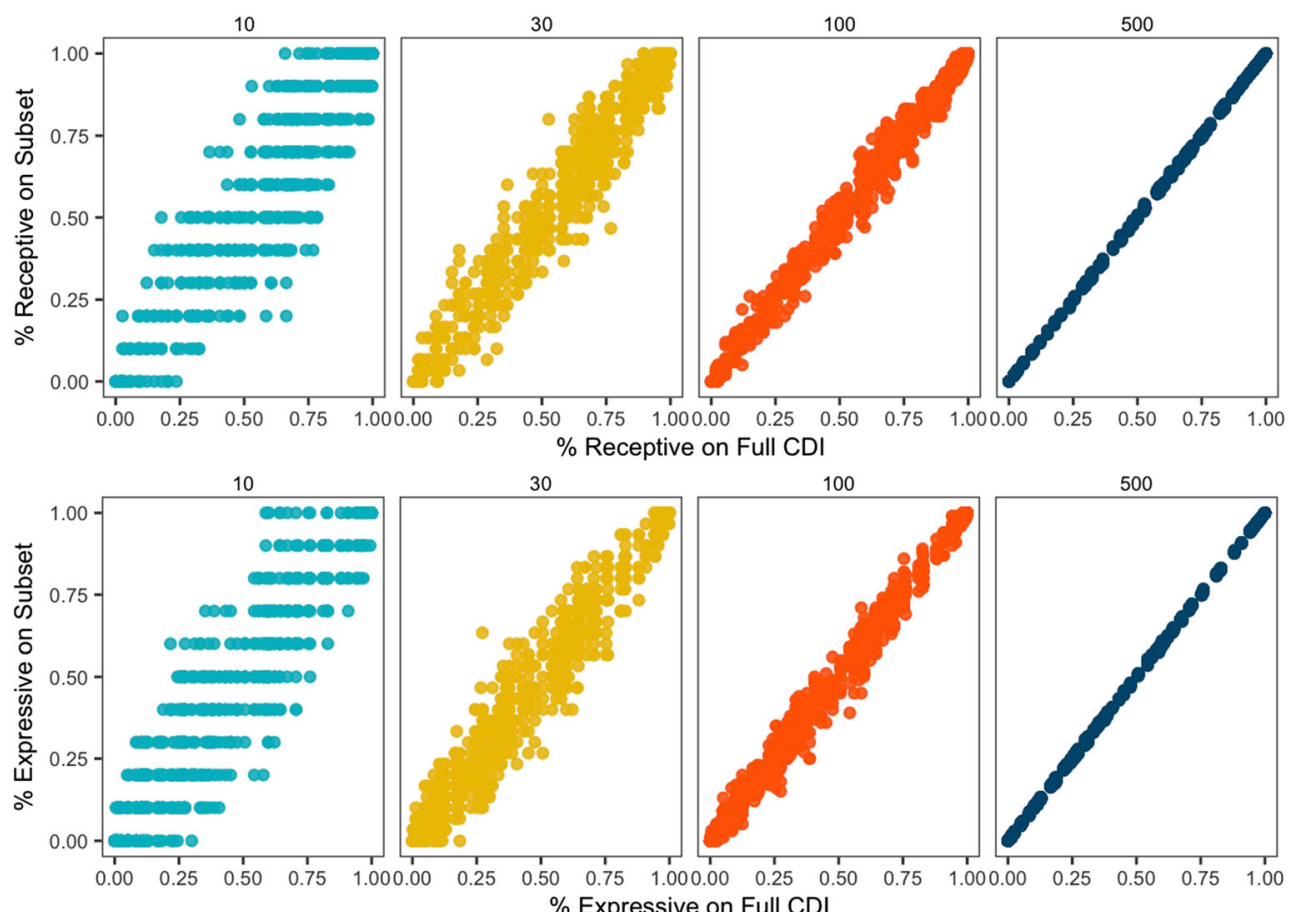

Fig. 10 The relationship between scores on the entire set of items on the ASL-CDI 2.0 and a randomly sampled subset of 10, 30, 100, and 500 words for both receptive and expressive vocabulary 
Unlike the MB-CDI, which has separate forms for younger (Words and Gestures) and older (Words and Sentences) children, the ASL-CDI is designed for a wide age range. The Vocabulary section and the Gestures and Phrases section are complementary: the Gestures and Phrases section is most sensitive among the youngest children, who may know very few or no signs. There is little variability in both expressive and receptive vocabulary size among the youngest children. Children in the normative sample reach ceiling by around 40 months for Receptive Vocabulary, 30 months for Gestures and Phrases, and 60 months for Expressive Vocabulary.

The primary findings of the ASL-CDI 1.0 were all replicated in the ASL-CDI 2.0 data set. Despite the fact that the ASL-CDI 1.0 was administered via English glosses and not ASL signs, the patterns observed with the ASL-CDI 1.0 and ASL-CDI 2.0 are very similar. Our replication thus reaffirms the validity of the CDI as a useful measure of ASL vocabulary production in deaf children.

At the same time, the ASL-CDI 2.0 extends the previous tool in several important ways. First, we now have sufficient data to estimate the expected vocabulary sizes for children across the age range. This information is necessary in order to use the tool in a clinical capacity. Additionally, the ASL-CDI 2.0 includes data on both receptive and expressive vocabulary. Receptive vocabulary is a critical marker of early communicative milestones. In particular, for the majority of deaf children who are not exposed to ASL from birth, receptive vocabulary is the natural starting point for measuring acquisition. The ASLCDI 2.0 also includes a Gestures and Phrases section, which is more sensitive to early differences in language acquisition and thus may be better suited to very young children than the Vocabulary section of the assessment alone.

Second, the ASL-CDI 2.0 extends the analysis of vocabulary composition. The current data set introduces an analysis of predicted and actual proportion of signs in each semantic category. The analyses here also show that the ASL-CDI 2.0 corresponds well to patterns found cross-linguistically that had not been demonstrated with the first adaptation. As expected, receptive vocabularies outpace expressive vocabularies. As has been found cross-linguistically, some semantic categories are overrepresented in children's vocabularies (animals, toys, and vehicles) and others are underrepresented (signs about time; Frank et al., 2019).

The ASL-CDI 2.0 illuminates the composition of early vocabulary with respect to the proportion of nouns and predicates. A number of studies have demonstrated that in several languages, nouns are learned more easily than other lexical classes (Bates et al., 1994; Bornstein et al., 2004; Gentner \& Boroditsky, 2009; Goldfield, 2000). Like Anderson and Reilly (2002), we see a slight noun bias in children's early vocabularies that decreases with age as children learn more predicates.

We offer a picture of the language acquisition trajectory of children acquiring ASL under optimal (i.e., not language- deprived) conditions, and with this in hand, the ASL-CDI 2.0 can be used to identify children who are falling behind in language acquisition. The ASL-CDI can be used both with children who have had limited exposure to ASL during early childhood, and children who may have additional diagnoses such as autism spectrum disorder or developmental language disorder. Children who fall below the average range may need additional support to acquire language. Note that a child who falls within the average range on this assessment may still have a language delay in other aspects of language development like syntax (e.g., Cheng \& Mayberry, 2019) or pragmatics (see Toe et al., 2016 for a review). Indeed, vocabulary appears to be one area in which children can catch up, while other areas are more resistant to intervention (Ferjan Ramirez, Lieberman, \& Mayberry, 2013; Thal, Tobias, \& Morrison, 1991).

The sample in this paper is small relative to the normative samples used in other adaptations of the CDI. The population of deaf children is small and geographically dispersed. To maximize the usefulness of this tool in detecting language delays in native signing children and language deprivation among deaf children exposed late to a sign language, we need norms from the very small subset of deaf children acquiring ASL natively (approximately $5-10 \%$ of deaf children in the U.S. and Canada). The sample size used here is two and three times the size of the other sign language adaptations of the CDI (Anderson \& Reilly, 2002; Woolfe et al., 2010).

There are a handful of caveats for using the assessment. In this study, we examined parents' ability to reliably complete the ASL-CDI 2.0, which is how other adaptations of the CDI are generally administered. Depending on a child's circumstances, there may be another person in the child's life who is better equipped to report on the child's vocabulary (e.g., a childcare provider who is more fluent in ASL than a parent and spends a significant amount of time with the child). We have not evaluated the accuracy of out-of-home caregivers in completing the ASL-CDI 2.0 and cannot provide any assessment about the measure's validity under these circumstances. In addition, some parents who are concerned about their child's language acquisition could conceivably misinterpret the ASL-CDI 2.0 as the entire set of signs that their child should know, rather than a set of signs that children may or may not know depending on age. As such, some parents may focus vocabulary instruction on the signs presented in this assessment, which may compromise the validity of repeat assessments. The use of the short form might mitigate this risk. Finally, vocabulary size can vary across children of the same age (e.g., a 20-month-old with a "typical" sized vocabulary could know anywhere from $16 \%$ to $68 \%$ of the signs). Users of the assessment should be cautioned not to interpret a "small" vocabulary size as necessarily indicative of a delay.

The vocabulary acquisition patterns we describe here are based on data from deaf children who received ASL in the first months of life. Because these children are exposed to fluent 
ASL users from birth, they are not at risk for language deprivation. How might the patterns of vocabulary development extend to deaf children with hearing parents? The language experience of deaf children with hearing parents is variable, and we consider two language scenarios for these children. First, a subset of deaf children of hearing parents are exposed to ASL immediately upon a diagnosis of deafness; they have early exposure to ASL, but the quantity and quality of input from parents learning ASL as a second language may be different than that from fluent signing deaf parents. For these children, it is critical to determine whether non-native exposure from hearing parents is sufficient for children to acquire vocabulary on a typical timescale. Second, many deaf children are not exposed to ASL until some period of time after birth, meaning they may not have access to any language in their first months of life. Here, the question is whether children without early exposure to ASL at birth can catch up to their natively exposed peers with regard to vocabulary development. If so, is there a threshold or cut-off for initial exposure age - as the critical period effect would suggest - after which vocabulary development is significantly delayed or different? More work is needed to characterize vocabulary development among deaf children with limited ASL exposure. The data from our validation study indicates that signing hearing parents can reliably report their deaf child's signed vocabulary, so the ASL-CDI 2.0 could be a useful tool for addressing these two questions.

Early vocabulary predicts other aspects of language development (e.g., syntax, pragmatics). Across spoken languages, it is correlated with many aspects of language acquisition (Braginsky, Yurovsky, Marchman \& Frank, 2019; Lee, 2011; McGregor, Sheng, \& Smith, 2005; Rowe, Raudenbush, \& Goldin-Meadow, 2012), and school-age ASL vocabulary abilities are correlated with other aspects of ASL proficiency (Henner, 2016). At the same time, deaf children represent a heterogeneous language population. More work is needed to confirm these relationships given the diversity of their language experience, and to determine whether early sign vocabulary correlates with other features of sign language development among children with language deprivation. With the ASL-CDI 2.0, we now have a tool to begin to address these questions.

\section{References}

Anderson, D., \& Reilly, J. (2002). The MacArthur communicative development inventory: normative data for American Sign Language. Journal of Deaf Studies and Deaf Education, 7(2), 83-106.

Bates, E., Marchman, V., Thal, D., Fenson, L., Dale, P., Reznick, J. S., ... \& Hartung, J. (1994). Developmental and stylistic variation in the composition of early vocabulary. Journal of Child Language, 21(1), $85-123$.
Bornstein, M. H., Cote, L. R., Maital, S., Painter, K., Park, S. Y., Pascual, L., ... \& Vyt, A. (2004). Cross-linguistic analysis of vocabulary in young children: Spanish, Dutch, French, Hebrew, Italian, Korean, and American English. Child Development, 75(4), 1115-1139.

Braginsky, M., Yurovsky, D., Marchman, V. A., \& Frank, M. C. (2019). Consistency and variability in children's word learning across languages. Open Mind, 3, 52-67. Chicago

Caselli, N. K., \& Pyers, J. E. (2017). The road to language learning is not entirely iconic: Iconicity, neighborhood density, and frequency facilitate acquisition of sign language. Psychological Science, 28(7), 979-987.

Caselli, N. K., \& Pyers, J. E. (2019). Degree and not type of iconicity affects sign language vocabulary acquisition. Journal of Experimental Psychology. Learning, Memory, and Cognition.

Caselli, N. K., Sehyr, Z. S., Cohen-Goldberg, A. M., \& Emmorey, K. (2017). ASL-LEX: A lexical database of American Sign Language. Behavior Research Methods, 49(2), 784-801.

Cheng, Q., \& Mayberry, R. I. (2019). Acquiring a first language in adolescence: the case of basic word order in American Sign Language. Journal of Child Language, 46(2), 214-240.

Dale, P. S., \& Fenson, L. (1996). Lexical development norms for young children. Behavior Research Methods, Instruments, \& Computers, 28(1), 125-127.

Duff, F. J., Reen, G., Plunkett, K., \& Nation, K. (2015). Do infant vocabulary skills predict school-age language and literacy outcomes? Journal of Child Psychology and Psychiatry, 56(8), 848-856.

Fenson, L., Dale, P. S., Reznick, J. S., Bates, E., Thal, D. J., Pethick, S. J., ... \& Stiles, J. (1994). Variability in early communicative development. Monographs of the society for research in child development, i-185.

Ferjan Ramírez, N., Lieberman, A. M., \& Mayberry, R. I. (2013). The initial stages of first-language acquisition begun in adolescence: when late looks early. Journal of child language, 40(2), 391-414.

Fieldsteel, Z., Bottoms, A., \& Lieberman, A. M. (accepted). Nouns and verbs in parent input in American Sign Language during interaction among deaf dyads. Language Learning and Development.

Frank, M. C., Braginsky, M., Yurovsky, D., \& Marchman, V. A. (2017). Wordbank: An open repository for developmental vocabulary data. Journal of child language, 44(3), 677-694.

Gentner, D., \& Boroditsky, L. (2009). Early acquisition of nouns and verbs: Evidence from Navajo. Routes to language: Studies in honor of Melissa Bowerman, 5-32.

Goldfield, B. A. (2000). Nouns before verbs in comprehension vs. production: the view from pragmatics. Journal of Child Language, 27(3), 501-520.

Hall, M. L., Eigsti, I. M., Bortfeld, H., \& Lillo-Martin, D. (2016). Auditory deprivation does not impair executive function, but language deprivation might: Evidence from a parent-report measure in deaf native signing children. The Journal of Deaf Studies and Deaf Education, 22(1), 9-21.

Hall, W. C. (2017). What you don't know can hurt you: The risk of language deprivation by impairing sign language development in deaf children. Maternal and hild health journal, 21(5), 961-965.

Hall, W. C., Levin, L. L., \& Anderson, M. L. (2017). Language deprivation syndrome: A possible neurodevelopmental disorder with sociocultural origins. Social Psychiatry and Psychiatric Epidemiology, 52(6), 761-776.

Hall, M. L., Hall, W. C., \& Caselli, N. K. (2019). Deaf children need language, not (just) speech. First Language, 0142723719834102.

Heilmann, J., Weismer, S. E., Evans, J., \& Hollar, C. (2005). Utility of the MacArthur-Bates Communicative Development Inventory in identifying language abilities of late-talking and typically developing toddlers. American Journal of Speech-Language Pathology, 14, $40-51$. 
Henner, J. (2016). The relationship between American Sign Language vocabulary and the development of language-based reasoning skills in deaf children (Doctoral dissertation, Boston University).

Henner, J., Novogrodsky, R., Reis, J., \& Hoffmeister, R. (2018). Recent issues in the use of signed language assessments for diagnosis of language disorders in signing deaf and hard of hearing children. The Journal of Deaf Studies and Deaf Education, 23(4), 307-316.

Lee, J. (2011). Size matters: Early vocabulary as a predictor of language and literacy competence. Applied Psycholinguistics, 32(01), 69-92.

Mayor, J., \& Mani, N. (2018). A short version of the MacArthur-Bates Communicative Development Inventories with high validity. Behavior Research Methods, 1-8.

McGregor, K., Sheng, L., \& Smith, B. (2005). The precocious two-yearold: Status of the lexicon and links to the grammar. Journal of Child Language, 32(03), 563-585.

Meir, N., Novogrodsky, R. (2018). Frequency and iconicity in ISL: Implications for acquisition. Talk presented at the Sign Language Acquisition and Assessment Conference, Haifa, Israel.

Mitchell, R. E., \& Karchmer, M. (2004). Chasing the mythical ten percent: Parental hearing status of deaf and hard of hearing students in the United States. Sign Language Studies, 4(2), 138-163.

Pyers, J. \& deVilliers, P. (2013). Theory of mind in deaf children: Illuminating the relative roles of language and executive functioning in the development of social cognition, in S. Baron-Cohen, H. Tager-Flusberg, \& M. Lombardo (Eds.) Understanding other Minds, 3rd Edition. Oxford University Press (345-363).

Rowe, M. L., Raudenbush, S. W., \& Goldin-Meadow, S. (2012). The pace of vocabulary growth helps predict later vocabulary skill. Child Development, 83(2), 508-525.

Sachse, S., \& Von Suchodoletz, W. (2008). Early identification of language delay by direct language assessment or parent report?. Journal of Developmental \& Behavioral Pediatrics, 29(1), 34- 41.

Simms, L., Baker, S., \& Clark, M. D. (2013). The standardized visual communication and sign language checklist for signing children. Sign Language Studies, 14(1), 101-124.

Sumer, B., Grabitz, C., \& Küntay, A. (2017). Early produced signs are iconic: Evidence from Turkish Sign Language. In the 39th Annual Conference of the Cognitive Science Society (pp. 3273-3278).

Thal, D., Tobias, S., \& Morrison, D. (1991). Language and gesture in late talkers: A 1-year follow-up. Journal of Speech, Language, and Hearing Research, 34(3), 604-612.

Thompson, R. L., Vinson, D. P., Woll, B., \& Vigliocco, G. (2012). The road to language learning is iconic: Evidence from British Sign Language. Psychological Science, 23(12), 1443-1448.
Toe, D., Rinaldi, P., Caselli, M. C., Paatsch, L., \& Church, A. (2016). The development of pragmatic skills in children and young people who are deaf and hard of hearing. In M. Marschark, V. Lampropoulou, \& E. Skordillis (Eds.) Diversity in Deaf Education. (pp. 247-269). Oxford: Oxford University Press.

Tonelson, S. (1980). A validation study of the Ski Hi language Development Scale, ProQuest Dissertations and Theses.

Woolfe, T., Herman, R., Roy, P., \& Woll, B. (2010). Early vocabulary development in deaf native signers: A British Sign Language adaptation of the communicative development inventories. Journal of Child Psychology and Psychiatry, 51(3), 322-331.

Publisher's note Springer Nature remains neutral with regard to jurisdictional claims in published maps and institutional affiliations.

Author Note First, thanks to Diane Anderson and Judy Reilly and Michael Frank for making the ASL-CDI 1.0 data available via WordBank. Thanks to Erin Spurgeon for organizing data collection, to Ethan Hartzell and Eric Alderman for helping create the parent portal, to Aiken Bottoms for contributing ASL versions of many questions and categories, and to Conrad Baer and Andrew Bottoms for creating ASL recruitment ads. Thank you to Carlisle Robinson for illustrations, and to Milana Donatich for help selecting the items. Thanks also to Jessica Korhumel, Kurt Gagne, Anna Lim Franck, Justin Bergeron, Brittany Farr, Michael Higgins, Tara Holcomb, Karianna Chamberlain, Alison Fitch, Deanna Gagne, Zoe Fieldsteel, Nicolette Pire, Megan Canne, Chelsea Hammond, and Julie Behar for their help preparing materials and in data collection. Thanks to Cindy O'Grady Farnady the use of her likeness, and to the parents and families who participated in this study. Research reported in this publication was supported by the National Institute on Deafness and Other Communication Disorders of the National Institutes of Health under Award Number R21DC016104 (NC) and Award Number R01DC015272 (AL). The content is solely the responsibility of the authors and does not necessarily represent the official views of the National Institutes of Health. This work is also supported by a James S. McDonnell Foundation Award to Jennie Pyers, and National Science Foundation grants BCS 1625793 and 1918252 to Naomi Caselli. 